\title{
An Investigation into noise-bound shadow detection and removal
}

\author{
K. Irie ${ }^{1}$, A.E. McKinnon ${ }^{2}$, K. Unsworth ${ }^{2}$, I.M. Woodhead ${ }^{1}$ \\ ${ }^{1}$ Lincoln Ventures Ltd, PO Box 133, Lincoln, Christchurch 7640, New Zealand. \\ ${ }^{2}$ Applied Computing Group, Lincoln University, Canterbury, New Zealand. \\ Email: iriek@lvl.co.nz
}

\begin{abstract}
Noise is an unavoidable contaminant in any non-trivial image. It is usually identified as a limiting factor in the performance of shadow-removal algorithms, but little is done to reduce its negative impact. The typical method to counter noise effects is to employ arbitrary or empirical thresholds somewhere inside the algorithm, with values chosen to maximize the shadow-removal performance. However these thresholds can be objectively calculated from the noise statistics for a particular pixel value. We present a method of shadow-removal whose internal parameters are adaptively set by noise statistics such that the algorithm is free of any empirically set threshold. Experiments indicate that the performance of the new method is approximately equivalent to that with an empirically-fixed threshold, though an area of improvement has been identified that could significantly boost the accuracy of the new method.
\end{abstract}

Keywords: noise, shadow removal, image analysis

\section{Introduction}

Noise is an unavoidable component of captured images. It is camera specific and is dependent on the number of photons arriving at the pixel capture site [1]. The signal-to-noise ratio is proportional to pixel value, with noise dominating in darker regions. Many image processing algorithms attempt to reduce the impact of image noise by using arbitrary or empirically set thresholds to segment true image data from noise [2-4] (often indirectly through the use of filters, such as threshold-based edge-detection routines). Others, such as in [5], use a statisticallycalculated fixed threshold. However, the image noise is treated as an additive component with no dependence upon pixel value, an assumption that results in sub-optimal performance of the algorithm, given the complexity of image noise [6].

In our previous work [4] we described a method for shadow-removal based upon the colour change of a pixel as the illuminant changes from daylight to skylight. Pixel changes were classified as being from cast shadows or from genuine changes in scene content. The classification process used set thresholds, empirically chosen to provide a balance between over-detection of noise and insensitivity to genuine scene change. In this work we attempt to replace this threshold with an adaptive and objectively calculated threshold value based upon the characteristics of the camera's noise. Section 2 summarises the shadow removal process in [4], with Section 3 describing the characteristics of measured noise for the camera used in our experiments. Section 4 describes the noise-bound shadow removal method, experimental results are given in section 5, and the paper closes with a discussion in section 6 .

\section{Dual-Illuminant Based Shadow Removal}

In [4] we describe a method for shadow removal based on pixel colour change over pairs of images. The method is summarised here.

The pixel response to illumination change is modelled, and then scene change detection is used to detect shadows. The pixel response, $P_{k}$, in an RGB camera is described by the product of illumination, surface reflectance, and camera sensitivities [7]:

$$
P_{k}=\int E(\lambda) S(\lambda) C_{k}(\lambda) d \lambda, \quad k \in\{R, G, B\}
$$

where $E$ is the illumination spectral power distribution (SPD), $S$ is the surface spectral reflectance function, $C$ is the camera response, $k$ is an RGB colour band of the camera, and $\lambda$ is the wavelength.

In outdoor conditions daylight and skylight have different SPDs. The change in pixel response can be modelled as the change in SPD from skylight to daylight:

$$
\begin{array}{r}
P_{k}=\int\left(E_{1}(\lambda)+n E_{2}(\lambda)\right) S(\lambda) C_{k}(\lambda) d \lambda, \\
k \in\{R, G, B\}, n \in[0,1]
\end{array}
$$


where $E_{1}$ is the ambient skylight source, $E_{2}$ is the directional sunlight source, and $n$ represents the proportion of added illumination $E_{2}$.

The use of the colour band ratios (also referred to as chromaticities) $x=R / G$ and $y=B / G$ provides a colour space that is intensity invariant. Taking the logs of the band ratios (LBR) as in [2] distributes the colour data relatively evenly enabling easier colour segmentation. The transform of the pixel response from RGB triplets to $x / y$ coordinates in LBR space is therefore

$$
x=\log \left(\frac{\int\left(E_{1}(\lambda)+n E_{2}(\lambda)\right) S(\lambda) C_{R}(\lambda) d \lambda}{\int\left(E_{1}(\lambda)+n E_{2}(\lambda)\right) S(\lambda) C_{G}(\lambda) d \lambda}\right)
$$

and

$$
y=\log \left(\frac{\int\left(E_{1}(\lambda)+n E_{2}(\lambda)\right) S(\lambda) C_{B}(\lambda) d \lambda}{\int\left(E_{1}(\lambda)+n E_{2}(\lambda)\right) S(\lambda) C_{G}(\lambda) d \lambda}\right)
$$

where $n \in[0,1]$. Equations (3) and (4) require the response in the camera's green channel to be nonzero. This is a reasonable expectation as the bandwidth of the colour filters in colour cameras is typically broad [8] and therefore the green channel would exhibit a response to almost any non-zero illumination in the visible spectrum.

A change in scene information can be described by any change in pixel response that does not fall on a curve in LBR space described by equation 2 as the illumination changes from $E_{1}+E_{2}$ to $E_{l}$. The addition of a region that bounds the modelled shadow curve accommodates the quantization of pixel values and the addition of image noise that may move measured values of $P_{k}$ away from the modelled curve in LBR space. This region is shown graphically in figure 1 .

We define $Q_{r e f}$ and $Q_{\text {comp }}$ as points in LBR space for a pixel from a reference and comparison image respectively. If $Q_{\text {comp }}$ falls inside the shadow region for $Q_{\text {ref }}$ then it is considered a shadow. A binary mask is created corresponding to each pixel in an image, where the mask value is zero if a pixel's $Q_{\text {comp }}$ falls inside $Q_{\text {ref }}$ 's shadow region otherwise it is set to one, indicating a genuine scene change in the scene. A shadow-free image is then created by combining the reference and comparison images from the mask, with zero-values being substituted by pixels in the reference image and one-values by pixels from the comparison image.

A Gretag-Macbeth (GMB) Color Chart was used to generate 19 shadow curves, one for each of its differently coloured panels (6 of the 24 panels are grey). The shadow curve for any pixel $Q_{\text {ref }}$ is approximated by the nearest-neighbour colour patch from the GMB chart calibration (in LBR space).

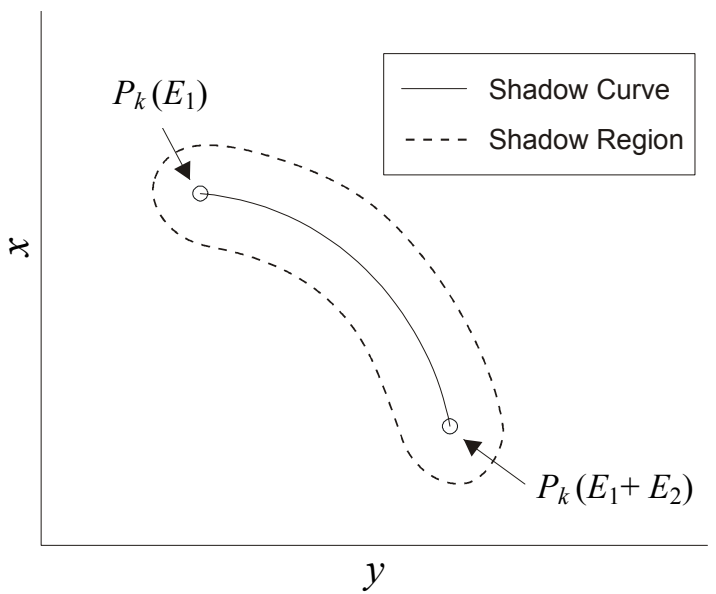

Figure 1: The modelled shadow curve in LBR space for a particular surface and associated shadow region for segmenting changes in a pixel response.

\section{Camera Noise}

In [6] we characterise and measure image noise for the $u E Y E$ CMOS camera (table 1). The resulting noise can be split into two components: temporal noise, which varies between images, and spatial noise, which varies across pixels in an image sensor. Our shadow-removal method compares pixel change between images, and hence only the temporal noise characteristics for the camera are required. The total temporal noise for the $u E Y E$ camera is shown in figure 2 , where $\mu_{G M B}$ panel is the mean value of the measured GMB panel and $\sigma_{\text {temp }}$ is the standard deviation of pixel variations over 100 images of the panel. These curves are used internally in our shadow removal algorithm to provide an objective means to segment shadows.

Table 1: $u E Y E$ UI1210-C camera details.

\begin{tabular}{|l|l|}
\hline Parameter & Value \\
\hline Sensor type & $1 / 2^{\prime \prime}$ CMOS (Bayer array) \\
\hline Native resolution & $640 \times 480$ \\
\hline Video mode & 24-bit RGB (8-bits/channel) \\
\hline Interface & USB 2.0 \\
\hline
\end{tabular}

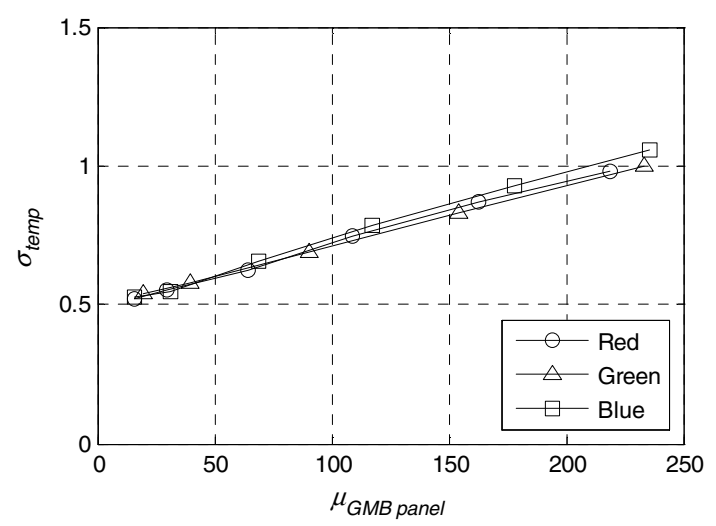

Figure 2: Total measured temporal noise for the CMOS camera described in table 1 , measured in a $22^{\circ} \mathrm{C}$ ambient environment. 


\section{Noise-Bound Shadow Removal}

The shadow-region threshold for each pixel in the reference image can be calculated based on its expected noise value given by the curves shown in figure 2. From (3) and (4), a pixel's $Q_{\text {ref }}$ has minimum and maximum noise boundaries in the LBR space that can be described by:

$$
\begin{aligned}
& x_{\text {max }}=\log \left(\frac{R+m \sigma_{R}}{G-m \sigma_{G}}\right), \\
& x_{\text {min }}=\log \left(\frac{R-m \sigma_{R}}{G+m \sigma_{G}}\right), \\
& y_{\text {max }}=\log \left(\frac{B+m \sigma_{B}}{G-m \sigma_{G}}\right), \\
& y_{\text {min }}=\log \left(\frac{B-m \sigma_{B}}{G+m \sigma_{G}}\right),
\end{aligned}
$$

where $m$ is the multiplier defining the desired confidence interval for the noise thresholds, and $\sigma_{R}$, $\sigma_{G}$, and $\sigma_{B}$ the standard deviations of temporal noise for a pixel's $R, G$, and $B$ values. A value of $m=2$ was used to provide confidence intervals of approximately $95 \%$.

Using (5), for any given values of pixel noise and $G$ there exists a shadow region around the curve in LBR space. The measurement of shadow curves using the GMB chart was performed in controlled lighting conditions, with fluorescent tubes used as the ambient illuminant $\left(E_{l}\right)$, and halogen bulbs used as the direct illuminant $\left(E_{2}\right)$. Noise-bound shadow regions were calculated by selecting $G$ values and varying $R$ and $B$ for each of the 19 measured GMB curves.

Example noise-bound shadow regions for several fixed $G$ values are shown in figure 3 for panel 13 of the GMB chart. High values of $G$ have small shadow regions which monotonically increase in size as the value of $G$ reduces. As a given shadow region is always a subset of another region with a lower $G$ value, each shadow region can be overlaid to create a single composite mask that can be used for shadow thresholding.

Figure 4 illustrates the original fixed-threshold shadow regions (left) and the composite noise-bound shadow regions (right) for 3 of the GMB panels imaged with the $u E Y E$ camera, where the grey-level inside each noise-bound region represents the value of the reference pixel's $G$ value ( 0 is represented by black and 255 by white). These images show that the fixed-threshold regions are far from optimal as their shape and size should vary with $G$.

For a pixel change to be classified as a shadow its $Q_{\text {comp }}$ must lie in the noise-bound shadow region defined by the $G$ value of the reference image pixel.

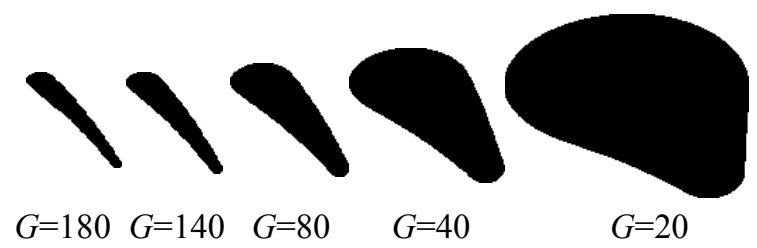

Figure 3: Example shadow regions associated with the calibrated panel 13 (purplish-blue) under controlled illumination conditions ( $x$ and $y$ axes removed for clarity). Each mask was generated with the indicated fixed $G$ value. Overlaying all of the valid masks between $G=0$ and $G=255$ results in the composite mask in figure $4 \mathrm{f}$.

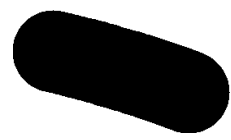

(a)

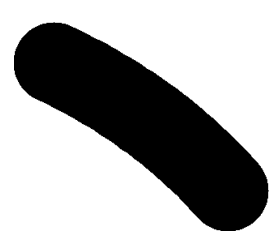

(c)

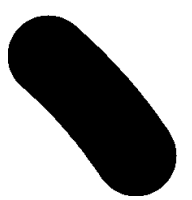

(e)

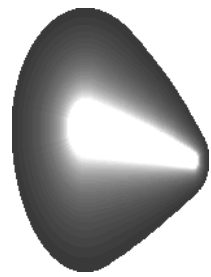

(b)

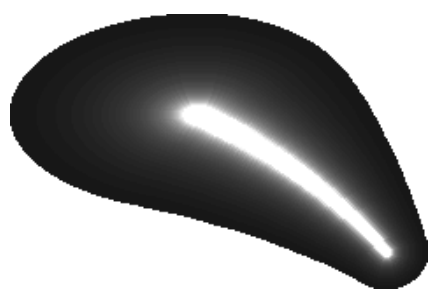

(d)

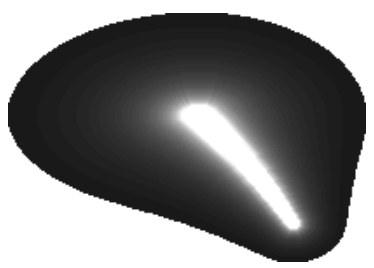

(f)
Figure 4: Original fixed-value shadow regions (left) and their equivalent noise-bound regions (right): (a) \& (b) GMB colour panel 15 (red), (c) \& (d) GMB colour panel 7 (orange), and (e) \& (f) GMB panel 13 (purplish-blue). Noise-bound regions scaled to 0-255 for clarity. Refer to figure 3 for generation of noisebound regions.

\section{Experimental Results}

The noise-bound shadow-removal method was tested on several shadowed images in controlled lighting conditions using the $u E Y E$ CMOS camera (table 1). 


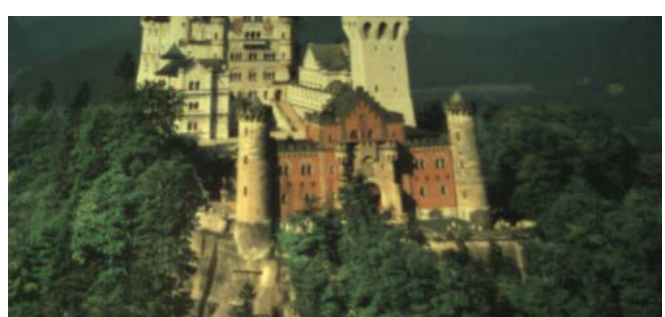

(a)

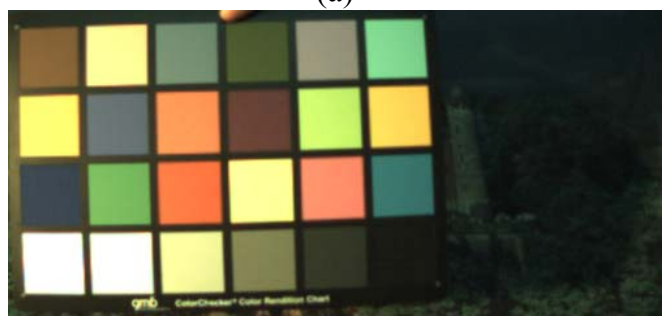

(b)

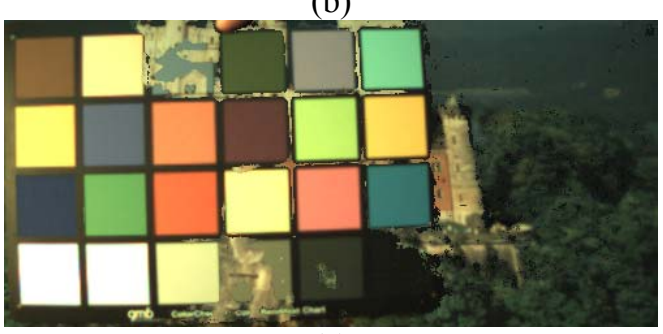

(c)

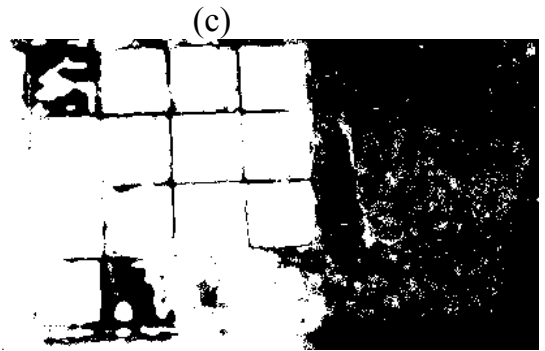

(d)

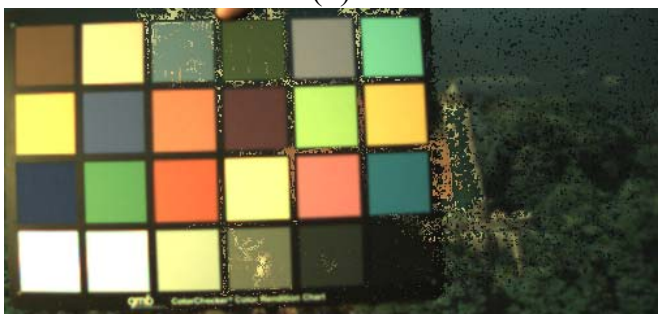

(e)

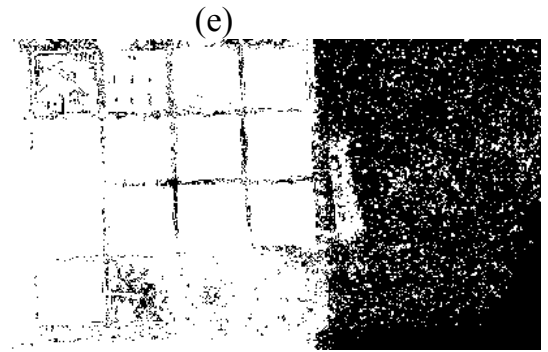

(f)

Figure 5: Shadow removal for a GMB chart in front of a background picture: (a) original image of picture, (b) image of GMB chart casting a shadow, (c) image after threshold-based shadow-removal and its mask of detected scene change (d), and (e) image after noisebased shadow-removal and its scene change mask (f).

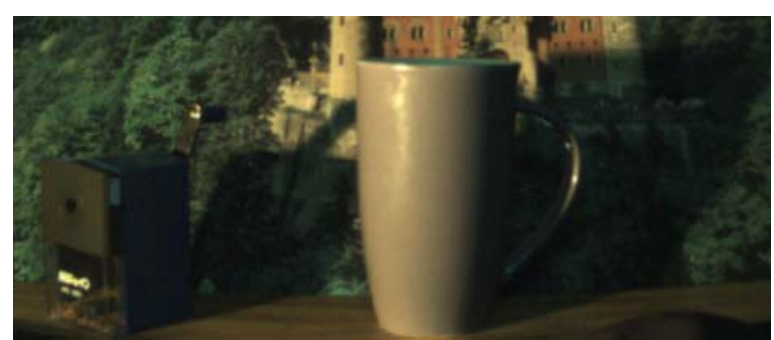

(a)

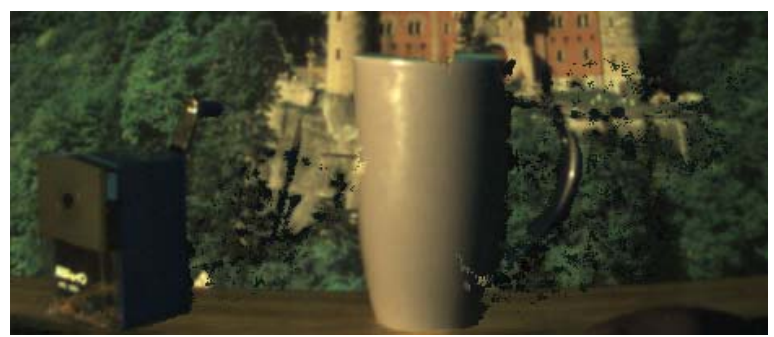

(b)

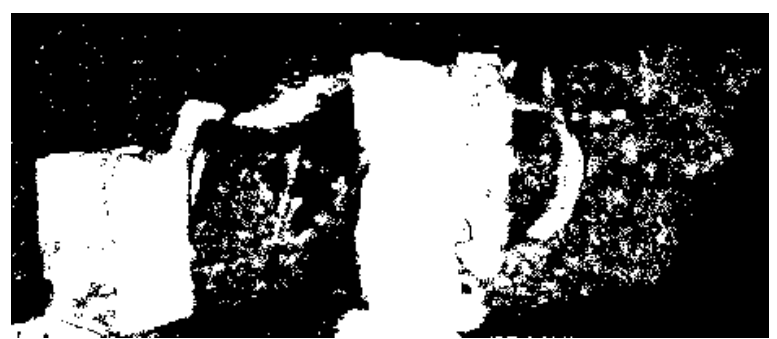

(c)

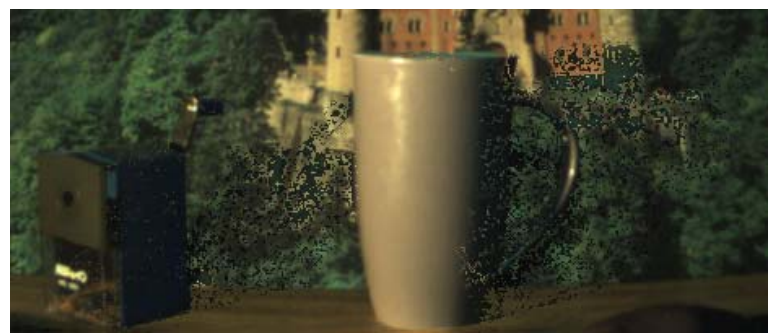

(d)

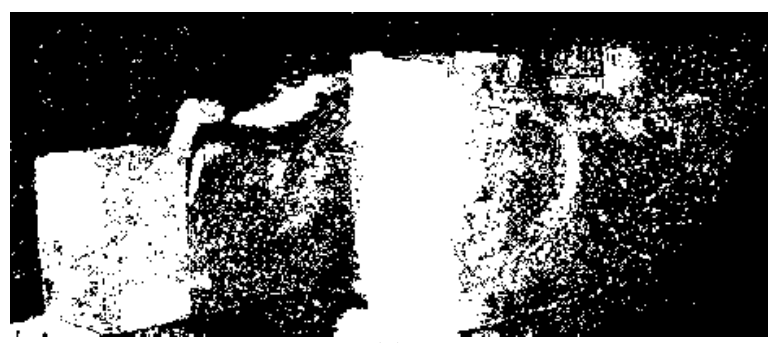

(e)

Figure 6: Shadow removal of a pencil sharpener and cup in front of the picture in 5a: (a) image with objects casting shadows, (b) image after thresholdbased shadow-removal and its mask of detected scene change (c), and (d) image after noise-based shadow removal and its scene change mask (e). 
Figures 5 and 6 show the results of the fixedthreshold and noise-bound shadow-removal methods. In both sequences a picture of a castle on a hill (figure $5 \mathrm{a}$ ) is used to provide a coloured and textured background for which to identify scene changes and cast shadows.

Figure $5 \mathrm{~b}$ shows the addition of the GMB chart with a strong cast-shadow on the right side of the image. A perfect shadow-removal algorithm would completely remove the shadow while leaving the GMB chart unchanged. The approach taken by the fixed-threshold method is to equally threshold all pixel colour changes away from the shadow curve in the log bandratio space, with the threshold empirically chosen so that the majority of shadowed pixels are removed, balanced against how much genuine scene change is incorrectly detected as shadow. The results are shown in figure $5 \mathrm{c}$ (the final shadow-removed image) and $5 \mathrm{~d}$ (the mask of detected scene change). The majority of the shadows are removed but consequently there are significant portions of the image which have been incorrectly detected as shadowed pixels, including panels 3 and 22 of the GMB chart and substantial portions of the black regions between and around the GMB colour panels. It is not unexpected that pixels with very low RGB values are classified as shadows, as the signal-to-noise ratio approaches unity making it difficult to distinguish black objects from dark shadows.

Figures $5 \mathrm{e}$ and $5 \mathrm{f}$ show the results from the noisebound shadow-removal algorithm. There are 3 substantial differences identified between the outputs of the two shadow removal methods: 1) the shadowremoved areas from the noise-bound algorithm shows more speckle than the fixed-threshold algorithm, which tends to have more clumpy regions of misclassification. 2) The integrity of the GMB chart is improved when the noise-bound algorithm is used. Panels 3, 22, and the black regions around the panels are better detected, though there is minor speckle in some parts of the objects. 3) The right-hand column of the castle has been incorrectly detected as shadow by the noise-bound algorithm. Similar results can be seen in the images in figure 6.

The large areas of castle that were incorrectly determined as shadows by the noise-bound algorithm were analysed further, with similar results shown in other experiments. Figure 7 illustrates an example where a shadow is cast over a coloured cloth background. The fixed-threshold method eliminates shadows from below the elephant, but leaves clumpy areas of misdetection on the elephant. The noisebound method shows an improvement in the removal of shadows on the elephant, but poorly removes the shadow from the area underneath the elephant. We hypothesise that the errors in the noise-bound shadow algorithm can be partially attributed to the limited number of measured shadow curves obtained from the GMB chart, where every pixel's shadow-curve is required to be approximated by one of the 19 differently colour panels on the chart. For example, a pixel in the reference image whose shadow-curve is between those of figures $4 \mathrm{c}$ and $4 \mathrm{e}$ would have a reasonable chance of successful shadow detection if either of the fixed-threshold figure $4 \mathrm{c}$ and $4 \mathrm{e}$ regions were chosen as the estimate for the pixel's true shadow-calibration curve, as both curves are similar in shape and orientation. However, the curves for the noise-bound method in figures $4 \mathrm{~d}$ and $4 \mathrm{f}$ show substantial levels of overlap only for low intensity pixel values and very little overlap for high intensity pixel values (white regions of figures $4 \mathrm{~d}$ and $4 \mathrm{f}$ ). Therefore any high-intensity pixels whose true shadow curve region lies between those of figure $4 d$ and $4 \mathrm{f}$ would receive a poor shadow curve estimate if they were the two nearest-neighbour curves, resulting in a high likelihood of error. This effect appears to provide a reasonable explanation for the errors in shadow detection in the walled area of the castle in figure $5 \mathrm{a}$ and in the cream background in figure $7 \mathrm{a}$, as both are relatively light in terms of pixel intensity. In both instances $Q_{\text {comp }}$ fell inside the fixed-threshold shadow region, but outside of the noise-bound shadow-region.

Overall, the performances of both shadow removal algorithms are similar, which validates the noisebound and threshold-free method as a potential alternative to the fixed-threshold method, even with the limitations described.

\section{Discussion}

A method for noise-bound shadow removal has been developed that is free from any empirical internal threshold. The statistically generated shadow-regions show that using a simple fixed-threshold boundary is sub-optimal with the size and shape of the regions varying with the specific colour and intensity of the reference pixel. However, experiments using noisebound regions exposed an apparent deficiency in the limited number of measured shadow curves used in our method. In the original fixed-threshold algorithm, small changes in the angle and shape of the curve caused by the nearest-neighbour curve-selection were hidden by the large radius of the threshold. The noisebound regions often closely hugged the shadow curve, especially for higher intensity pixels, which resulted in sensitivity to errors in the shadow curve approximation.

Further research is continuing in the generation of a greater number of shadow curves, which is expected to improve the performance of the noise-bound shadow removal method. 


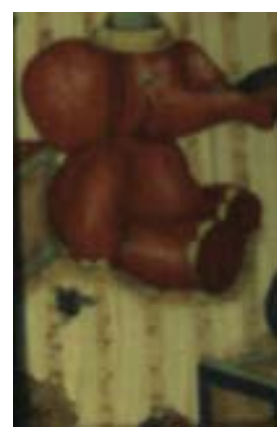

(a)

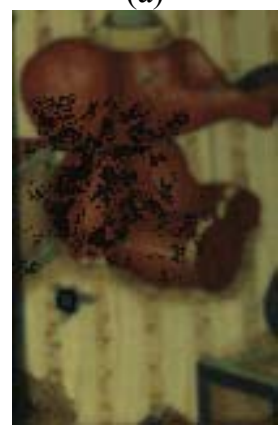

(c)

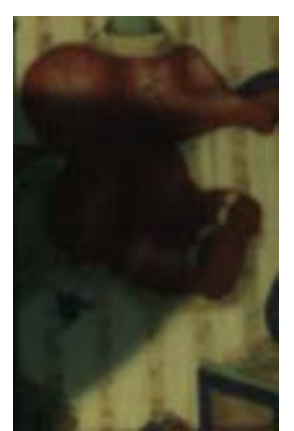

(b)

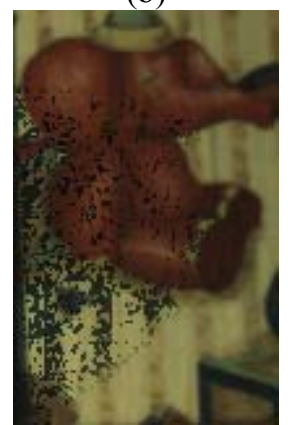

(d)
Figure 7: Example of differences in performance of the fixed-threshold and noise-bound shadow removal algorithms: (a) original image without shadow, (b) image with cast shadow, (c) shadowed image processed with fixed-threshold based algorithm, and (d) shadowed image processed with noise-bound algorithm.

\section{Acknowledgement}

This work was supported by the New Zealand Foundation for Research, Science and Technology programme LVLX0401.

\section{References}

[1] K. Irie, A. E. McKinnon, K. Unsworth, and I. M. Woodhead, "A model for evaluation of noise in CCD digital video cameras (accepted for publication)," Measurement, Science and Technology vol. 19, 2008.

[2] G. D. Finlayson, S. D. Hordley, C. Lu, and M. S. Drew, "On the removal of shadows from images," IEEE Trans. Pattern Analysis and Machine Intelligence, vol. 28, pp. 59-68, 2006.

[3] P. J. Withagen, F. C. A. Groen, and K. Schutte, "IAS technical report IAS-UVA-0702 Shadow detection using a physical basis," Intelligent Autonomous Systems, University of Amsterdam 2007.

[4] K. Irie, A. E. McKinnon, K. Unsworth, and I. M. Woodhead, "Shadow Removal for Object Tracking in Complex Outdoor Scenes," Proc. Image and Vision Computing New Zealand, pp. 25-29, 2007.
[5] A. Leone, C. Distante, and F. Buccolieri, "A shadow elimination approach in videosurveillance context," Pattern Recogn. Lett., vol. 27, pp. 345-355, 2006.

[6] K. Irie, A. E. McKinnon, K. Unsworth, and I. M. Woodhead, "Measurement of Digital Camera Image Noise for Imaging Applications," Sensors and Transducers, vol. 90, pp. 185-194, 2008.

[7] R. Gershon, A. D. Jepson, and J. K. Tsotsos, "From [R, G, B] to Surface Reflectance: Computing Color Constant Descriptors in Images," Proc. Int. Joint Conf. Artificial Intelligence, pp. 755-758, 1987.

[8] J. Nakamura, Image Sensors and Signal Processing for Digital Still Cameras: CRC Press, 2006. 